\title{
Image Processing Techniques for Acute Leukaemia Detection
}

\author{
Ashwini P. Patil ${ }^{1}$, Manjunatha Hiremath ${ }^{2}$ \\ ${ }^{1} \mathrm{PhD}$ Scholar, of Computer Science, CHRIST (Deemed to be University), Bengaluru, Assistant Professor, \\ Department of Computer Application, CMR Institute of Technology, Bengaluru, India, \\ patil.ashwini.p@gmail.com \\ ${ }^{2}$ Assistant Professor, Department of Computer Science,CHRIST(Deemed to be University), Bengaluru, India, \\ manjunatha.hiremath@christuniversity.in
}

\begin{abstract}
Every year a lot of people are diagnosed with blood cancer. This disease has a high mortality rate due to late and incorrect diagnoses. Acute leukaemia diagnosis requires an automated solution to facilitate early detection. This is one of the challenging problems and several machine learning techniques are proposed in recent years, however, image analysis using blood smear images remains the easiest and efficient technique to detect acute leukaemia. This study provides a literature review of the research work corresponding to the detection and classification of Acute lymphoblastic leukaemia (ALL) using digital image processing. The paper presents the analysis of various methods and techniques of image processing to detect leukaemia and highlights some of the research pros and cons. This literature review also introduces some of the research issues and challenges involved in this field of study
\end{abstract}

Key words : Acute lymphoblastic Leukemia, Classification, Image processing, Image segmentation..

\section{INTRODUCTION}

Leukaemia is one of the common blood cancers claiming many lives every year. Leukaemia is a blood cell cancer that originates in bone marrow spreading further to peripheral blood and other organs. Leukemic cancer can be classified into various types, based on the immaturity level of the cell it is divided as Acute or Chronic, and based on cell lineage it is divided into myeloid or lymphoid. Hence there are four different types of leukaemias i.e. Acute lymphoblastic leukaemia, Acute Myeloid leukaemia, Chronic lymphoblastic leukaemia, and Chronic Myeloid leukaemia. According to FAB Classification[1] two acute leukaemia are further divided into subcategories. Lymphoblastic leukaemia is subdivided into three types (L1, L2, and L3) whereas myeloid leukaemia is subdivided into six types (M1, M2, M3, M4, M5, and M6).

Acute leukaemia is considered riskier because it grows and spreads rapidly as compared to chronic and there would be very little time for diagnosis and treatment. Hence early detection of Acute leukaemia is very important. The diagnosis of leukaemia is done in three stages. First step is a complete blood count $(\mathrm{CBC})$ test which gives the total count of hemoglobin, white blood cells, red blood cells, platelets and hematocrit. CBC test is one of the easiest and cheapest but it gives a complete analysis of your blood and is the most effective one. The second step is bone marrow aspiration; this test further helps in confirmation of leukaemia. The third step is to classify the type of leukaemia by analyzing the cancer cells characteristics.

This complete leukaemia diagnosis needs good infrastructure and an expert pathologist which might be a problem for underdeveloped as well as developing countries. Moreover this process if done manually will take a lot of time and diagnosis might get delayed. Due to these delays in diagnosis most of the patients are at advanced disease (stage 3-4) at the time of their diagnosis [21]. There is certainly a need to develop a completely automated, cost-efficient system to diagnose patients with leukaemia and detect the type of leukaemia.

A lot of studies have been done to find a better solution for leukaemia detection. Image processing using blood smear images serves as an efficient technique for early diagnosis of leukaemia. This paper reviews some of the studies and research work done in this area. The paper is organized as follows: Section1 defines the study; Section2 discusses the review protocol; Section3 gives details of the dataset; Section 4 presents the review of methodology and summary of all the methods and techniques extracted from review papers; Section5 specify the research issues Section6 provides the conclusion.

\section{DATASET}

The first step in the detection of leukaemia disease is the acquisition of peripheral blood images. Most of the studies in this survey have used publicly available ALL-IDB database. The ALL-IDB dataset has two different versions (ALL-IDB1 and ALL-IDB2)[1]. Some of the studies which focus on ALL and AML classification have acquired images from The American Society of Hematology (ASH) Image Bank. ASH 
Ashwini P. Patil et al., International Journal of Emerging Trends in Engineering Research, 8(10), October 2020, 7320 - 7327

bank is a web-based image library containing a wide range of hematology images [2]. Some of the articles have collected a dataset from the local hospitals and pathology centres. Fig.1 shows an example of peripheral blood microscopic images. Table 1 provides a summary of acute leukaemia datasets used in reviewed research papers.

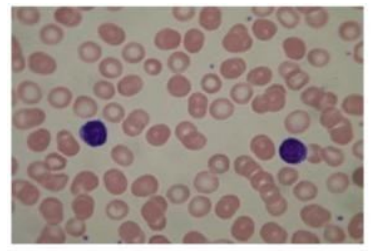

(a)

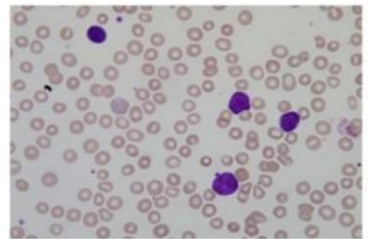

(b)

Figure 1: Examples of images from ALL- IDB: (a) Healthy Blood (b) Blood with ALL blast

Table 1: Datasets used in reviewed research

\begin{tabular}{|c|c|c|c|c|}
\hline \# & Ref & Dataset & Number of Samples & Sources \\
\hline 1 & [6] & Private Dataset & $\begin{array}{c}100 \text { microscopic images } \\
\text { (Training } 62 \text { images, Testing } 38 \text { images) }\end{array}$ & $\begin{array}{c}\text { Medical Image and Signal } \\
\text { Processing Research Centre (MISP) }\end{array}$ \\
\hline 2 & $\begin{array}{c}{[15][17]} \\
{[19]}\end{array}$ & Public Dataset & $\begin{array}{l}108 \text { microscopic images } \\
\text { Of resolution }(2592 \times 1944)\end{array}$ & ALL-IDB \\
\hline 3 & [3] [4] & Public Dataset & - & ALL-IDB2 \\
\hline 4 & [18] & Public Dataset & $\begin{array}{c}240 \text { microscopic images } \\
(100 \text { - healthy, } 80 \text { - AML, } 60 \text { - ALL) }\end{array}$ & $\begin{array}{l}\text { American society of hematology } \\
\text { (ASH) Image Bank }\end{array}$ \\
\hline 5 & [9] & Public Dataset & $\begin{array}{c}80 \text { single cell images of } 60 \times 60 \text { pixels } \\
(40-\text { normal blood cells, } \\
40-\text { abnormal blood cells })\end{array}$ & ALL-IDB \\
\hline 6 & [8] & $\begin{array}{l}\text { Public Dataset and } \\
\text { Private Dataset }\end{array}$ & $\begin{array}{l}14 \text { images }(2864 \times 2909 \text { pixel }) \\
59 \text { images }(2592 \times 1944 \text { pixel }) \\
215 \text { images }(300 \times 300 \text { pixel }) \\
\end{array}$ & $\begin{array}{c}\text { Cellavision database } \\
\text { ALL-IDB database } \\
\text { Jiashan database }\end{array}$ \\
\hline 7 & [5] & Public Dataset & - & ALL-IDB1 \\
\hline 8 & [7] & Private Dataset & - & $\begin{array}{c}\text { Ispat General Hospital, Rourkela } \\
\text { Orissa and } \\
\text { University of Virginia }\end{array}$ \\
\hline 9 & [13] & Private Dataset & $\begin{array}{l}1500(750 \mathrm{AML}+750 \text { ALL }) \text { Images } \\
\text { Training } 1200(600 \mathrm{AML}+600 \mathrm{ALL}) \text { images, } \\
\text { Testing } 300(150 \mathrm{AML}+150 \mathrm{ALL}) \text { images }\end{array}$ & $\begin{array}{c}\text { Hematology Department at } \\
\text { University Sains Malaysia Hospital } \\
\text { (HUSM) in Kubang Kerian, } \\
\text { Kelantan, Malaysia }\end{array}$ \\
\hline 10 & [14] & $\begin{array}{l}\text { Public Dataset and } \\
\text { Private Dataset }\end{array}$ & $\begin{array}{c}115 \text { public images }(632 \times 480 \text { pixel }) \\
642 \text { private images }\end{array}$ & ALL-IDB \\
\hline 11 & [16] & Public Dataset & 260 images of resolution $(257 \times 257)$ pixel & ALL-IDB \\
\hline 12 & [11] & Public Dataset & 33 microscopic images & ALL-IDB1 \\
\hline 13 & [20] & Public Dataset & $\begin{array}{l}15 \text { testing and } 130 \text { training images for cell } \\
\text { counting } \\
85 \text { Images (1712 x } 1368 \text { pixels) for detection }\end{array}$ & ALL-IDB \\
\hline
\end{tabular}

\section{REVIEW OF METHODOLOGY}

Medical image processing is an analysis technique in which we apply machine learning to automate medical diagnosis. Image processing in leukaemia diagnosis can be broadly classified into four stages, Pre-processing, segmentation, feature extraction and classification. Figure 2 shows the detailed workflow of all the stages involved in acute leukaemia detection. This section reviews all the methods used in selected research articles. Table 2 summarizes the comparison of all the methodologies adopted by reviewed articles.

\subsection{Pre-processing}

Image pre-processing includes image restoration, transformation, filtering and enhancement. Images have to be pre-processed before feeding them into the detection system because it greatly impacts segmentation and feature extraction steps. In pre-processing [19] converts RGB image into a grayscale image, but most of the researchers propose that converting RGB model to other colour space like YCBR[6], CY[4], CMYK[17] is more effective. [14] proves that selecting only a green component from an RGB image can also result in better accuracy in segmentation. 
$[11,12,15,17,18,19]$ have used histogram equalization to improve image contrast. Some of the articles have used various filtering techniques such as Order statistic filter [18], wiener filtering [15, 10], Selective filtering [7] etc.

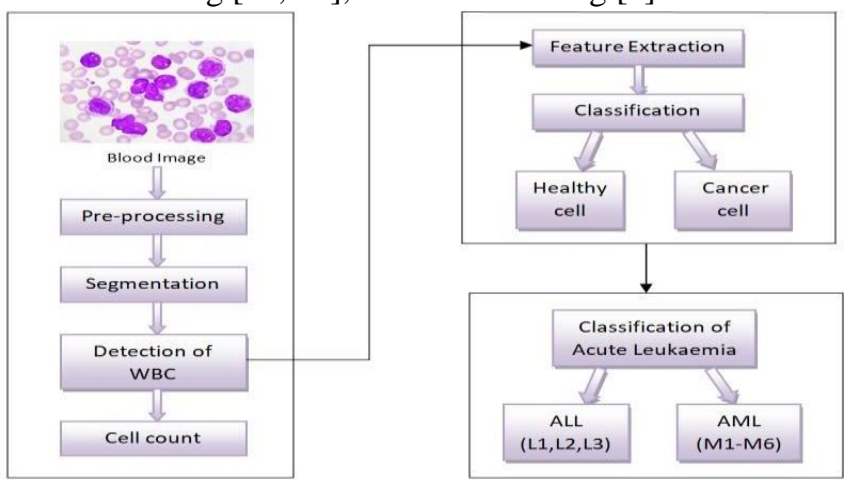

Figure 2: Stages of Acute Leukemia Detection using Image Processing

\subsection{Segmentation}

Image segmentation is one of the crucial steps in leukaemia detection.

Lot of research work has been carried out in segmentation techniques and it is still one of the challenging steps in the entire process. In this step, the blast cell is identified and then nucleus and cytoplasm are segmented. Most common techniques used in this step are watershed algorithms, Otsu's thresholding and morphological operations. [9, 15] have proposed a novel marker-based segmentation (MBS) method for segmentation. [13] shows that the segmentation step could be excluded thus reducing computational and time cost.

\subsection{Feature Extraction}

In feature extracting and selection we extract desired features to identify or classify objects. Some of the common features that are used in leukaemia detection are colour, texture, contour, chromatic, geometric and statistical features.

\subsection{Classification}

Selected features from the previous steps are fed to the classification algorithm to detect leukaemia blast cells and healthy cells and further classify them into subcategories. The accuracy of classification largely depends upon features extracted and used to train the algorithms. Random forest, neural network, support vector machine (SVM), decision tree and k-nearest neighbour are the common methods used in leukaemia detection and classification.

Table 2: Comparison of reviewed literature

\begin{tabular}{|c|c|c|c|c|c|}
\hline $\begin{array}{l}\text { Author } \\
\text {, year }\end{array}$ & Proposed work & Methods & & Merits and Demerits & Results \\
\hline \multirow{4}{*}{$\frac{\frac{\text { Jha \& }}{\text { Dutta, }}}{\frac{2019}{[3]}}$} & \multirow{4}{*}{$\begin{array}{l}\text { Detection of } \\
\text { Acute } \\
\text { Lymphocytic } \\
\text { Leukemia using } \\
\text { Mutual } \\
\text { Information } \\
\text { based hybrid } \\
\text { model and deep } \\
\text { learning }\end{array}$} & Preprocessing & Image Resizing & \multirow{4}{*}{$\begin{array}{l}\text { Merits: } \\
\text { Proposed two novel } \\
\text { approaches } \\
\text { 1) MI based hybrid model } \\
\text { used for segmentation } \\
\text { 2)Chronological SCA based } \\
\text { deep CNN classifier }\end{array}$} & \multirow{4}{*}{$98.7 \%$ Accuracy } \\
\hline & & Segmentation & $\begin{array}{l}\text { Mutual Information(MI) } \\
\text { based hybrid model which } \\
\text { employs Active contour } \\
\text { and FCM based image } \\
\text { Segmentation }\end{array}$ & & \\
\hline & & $\begin{array}{l}\text { Feature } \\
\text { Extraction }\end{array}$ & $\begin{array}{l}\text { Color histogram features, } \\
\text { LDP features }\end{array}$ & & \\
\hline & & Classification & $\begin{array}{l}\text { Chronological Sine } \\
\text { Cosine Algorithm (SCA) } \\
\text { based Deep CNN }\end{array}$ & & \\
\hline \multirow{2}{*}{$\begin{array}{l}\frac{\text { Moham }}{\text { med et }} \\
\frac{\text { al., }}{2014} \\
{[4]}\end{array}$} & \multirow{2}{*}{$\begin{array}{l}\text { Acute } \\
\text { Lymphoblastic } \\
\text { Leukemia } \\
\text { Segmentation } \\
\text { Using C-Y Color } \\
\text { Space }\end{array}$} & Preprocessing & $\begin{array}{l}\text { Convert image to CY color } \\
\text { model, Extract Y } \\
\text { component }\end{array}$ & \multirow{2}{*}{$\begin{array}{l}\text { Merits: } \\
\text { By applying CY color space } \\
\text { achieved better accuracy as } \\
\text { compared to RGB color } \\
\text { space }\end{array}$} & \multirow[b]{2}{*}{ Segmentation accuracy is $98.38 \%$} \\
\hline & & Segmentation & $\begin{array}{l}\text { Use histogram, median } \\
\text { filter, convert final image } \\
\text { to RGB }\end{array}$ & & \\
\hline \multirow{3}{*}{$\begin{array}{l}\frac{\text { Bhattac }}{\text { harjee }} \\
\frac{\text { \& Saini, }}{\frac{2015}{[5]}}\end{array}$} & \multirow{3}{*}{$\begin{array}{l}\text { Compare four } \\
\text { types of } \\
\text { classifiers and } \\
\text { develop } \\
\text { graphical user } \\
\text { interface using } \\
\text { classifier with }\end{array}$} & Preprocessing & $\begin{array}{l}\text { Contrast enhancement and } \\
\text { Quality adjustment }\end{array}$ & \multirow{3}{*}{$\begin{array}{l}\text { Merits } \\
\text { 1)Achieved better } \\
\text { segmentation accuracy } \\
\text { using morphological } \\
\text { operations as compared to } \\
\text { other techniques } \\
\text { 2)developed GUI for }\end{array}$} & \multirow{3}{*}{ Segmentation accuracy is $96.67 \%$} \\
\hline & & Segmentation & $\begin{array}{l}\text { Morphological Dilation } \\
\text { and Erosion }\end{array}$ & & \\
\hline & & $\begin{array}{l}\text { Feature } \\
\text { Extraction }\end{array}$ & $\begin{array}{l}\text { Morphological } \\
\text { features(Area, Perimeter, } \\
\text { circularity, form factor) }\end{array}$ & & \\
\hline
\end{tabular}


Ashwini P. Patil et al., International Journal of Emerging Trends in Engineering Research, 8(10), October 2020, 7320 - 7327

\begin{tabular}{|c|c|c|c|c|c|c|c|c|c|c|c|}
\hline & \multirow{8}{*}{$\begin{array}{l}\text { best output to } \\
\text { detect ALL }\end{array}$} & \multirow[t]{8}{*}{ Classification } & \multirow{8}{*}{$\begin{array}{l}\text { KNN, SVM, ANN, } \\
\text { K-means clustering }\end{array}$} & \multirow{8}{*}{$\begin{array}{l}\text { segmentation and } \\
\text { classification of lymphoid } \\
\text { cells } \\
\underline{\text { Demerits }} \\
\text { Lymphocytes in the image } \\
\text { are individuated manually }\end{array}$} & \multirow{2}{*}{\multicolumn{2}{|c|}{$\begin{array}{l}\text { Figs. of Merit } \\
\text { True Positive } \\
\end{array}$}} & kNN & & VM & ANN & K-means \\
\hline & & & & & & & & \begin{tabular}{l|l}
9 & \\
\end{tabular} & \begin{tabular}{c|c}
9 \\
\end{tabular} & 9 & \\
\hline & & & & & \multicolumn{2}{|c|}{ True Negative } & & 20 & 19 & 20 & 18 \\
\hline & & & & & \multirow{2}{*}{\multicolumn{2}{|c|}{ False Negative }} & & 1 & 2 & 1 & 3 \\
\hline & & & & & & & & 0 & 0 & 0 & 0 \\
\hline & & & & & \multicolumn{2}{|c|}{ Misclassification } & \multicolumn{2}{|c|}{$4.76 \%$} & $9.52 \%$ & $4.76 \%$ & \multirow{2}{*}{\begin{tabular}{|l|}
$14.28 \%$ \\
$85.71 \%$
\end{tabular}} \\
\hline & & & & & \multicolumn{2}{|c|}{ Specificity } & \multicolumn{2}{|c|}{$95.23 \%$} & $90.47 \% 9.5$ & $95.23 \%$ & \\
\hline & & & & & Sensitivit & & 100 & & $100 \%$ & $100 \%$ & $100 \%$ \\
\hline \multirow{5}{*}{$\frac{\frac{\text { Agrawa }}{\frac{\text { let al., }}{2019}}}{[6]}$} & \multirow{5}{*}{$\begin{array}{l}\text { To develop } \\
\text { automated } \\
\text { system for early } \\
\text { detection of } \\
\text { WBC cancer i.e. } \\
\text { Myeloma and } \\
\text { Leukemia (ALL } \\
\text { and AML) }\end{array}$} & Preprocessing & $\begin{array}{l}\text { Convert RGB image to } \\
\text { YCBCR color space }\end{array}$ & \multirow{5}{*}{$\begin{array}{l}\text { Merits } \\
\text { 1) Detects Myeloma and } \\
\text { types of Leukemia } \\
\text { 2) This technique requires } \\
\text { minimum preprocessing and } \\
\text { excellent feature extraction }\end{array}$} & \multirow{5}{*}{\multicolumn{7}{|c|}{$\begin{array}{l}\text { Detect ALL, AML and Myeloma } \\
\text { with } 97.3 \% \text { Accuracy }\end{array}$}} \\
\hline & & Segmentation & Gaussian Distribution, & & & & & & & & \\
\hline & & & $\begin{array}{l}\text { Otsu's Thresholding, } \\
\text { Kmeans clustering }\end{array}$ & & & & & & & & \\
\hline & & $\begin{array}{l}\text { Feature } \\
\text { Extraction }\end{array}$ & $\begin{array}{l}\text { GLCM method to extract } \\
\text { texture features. }\end{array}$ & & & & & & & & \\
\hline & & Classification & $\mathrm{CNN}$ & & & & & & & & \\
\hline \multirow[t]{4}{*}{$\begin{array}{l}\text { Joshi et } \\
\text { al..2013 } \\
{[19]}\end{array}$} & \multirow{4}{*}{$\begin{array}{l}\text { This work } \\
\text { propose } \\
\text { automatic WBC } \\
\text { segmentation } \\
\text { and classifyblast } \\
\text { cells from } \\
\text { normal } \\
\text { lymphocyte cells }\end{array}$} & Preprocessing & $\begin{array}{l}\text { Convert image into gray } \\
\text { scale image, use } \\
\text { histogram equalization, } \\
\text { linear contrast stretching } \\
\text { to adjust intensity of } \\
\text { image }\end{array}$ & \multirow{4}{*}{$\begin{array}{l}\frac{\text { Merits }}{\text { Good accuracy with }} \\
\text { minimal feature extraction } \\
\frac{\text { Demerits }}{\text { Only classify blast cells and }} \\
\text { normal cell, does not detect } \\
\text { type of leukemia }\end{array}$} & \multirow{4}{*}{\multicolumn{7}{|c|}{ 93\% Accuracy }} \\
\hline & & Segmentation & $\begin{array}{l}\text { Otsu's Thresholding, } \\
\text { morphological opening, } \\
\text { size test removal to } \\
\text { discard unwanted objects }\end{array}$ & & & & & & & & \\
\hline & & $\begin{array}{l}\text { Feature } \\
\text { Extraction }\end{array}$ & $\begin{array}{l}\text { Shape feature (area, } \\
\text { perimeter and circularity) }\end{array}$ & & & & & & & & \\
\hline & & Classification & KNN & & & & & & & & \\
\hline \multirow{4}{*}{$\begin{array}{l}\frac{\text { Mohapa }}{\text { tra et }} \\
\frac{\text { al.. }}{2010} \\
\frac{[7]}{[7]}\end{array}$} & \multirow{4}{*}{$\begin{array}{l}\text { Proposed work } \\
\text { to classify a } \\
\text { lymphocyte into } \\
\text { normal or a ALL } \\
\text { lymphoblast. }\end{array}$} & Preprocessing & $\begin{array}{l}\text { Selective filtering, } \\
\text { un-sharp masking, } \\
\text { RGB to Lab color space } \\
\end{array}$ & \multirow{4}{*}{$\begin{array}{l}\text { Merits } \\
\text { Two new features ie. } \\
\text { Contour signature and } \\
\text { hausdorff dimension are } \\
\text { used to classify lymphocyte } \\
\text { cell nucleus }\end{array}$} & \multirow{4}{*}{\multicolumn{7}{|c|}{$95 \%$ Accuracy }} \\
\hline & & Segmentation & FCM and Bounding box & & & & & & & & \\
\hline & & $\begin{array}{l}\text { Feature } \\
\text { Extraction }\end{array}$ & $\begin{array}{l}\text { Texture, color space, } \\
\text { Fractals by hausdorff } \\
\text { dimension, contour } \\
\text { signature }\end{array}$ & & & & & & & & \\
\hline & & Classification & SVM & & & & & & & & \\
\hline \multirow{4}{*}{$\begin{array}{l}\text { Zhao et } \\
\frac{\text { al.. }}{2017} \\
{[8]}\end{array}$} & & Preprocessing & R-B image & $\underline{\text { Demerits }}$ & & & & & & & \\
\hline & system to detect & Segmentation & Morphological operations & It fails to detect all the & & & & & & & \\
\hline & $\begin{array}{l}\text { and classify } \\
\text { WBC }{ }^{\text {ee }} \text { s from } \\
\text { peripheral blood } \\
\text { images }\end{array}$ & $\begin{array}{l}\text { Feature } \\
\text { Extraction }\end{array}$ & $\begin{array}{l}\text { Extract Granularity } \\
\text { features(PRICoLBP) from } \\
\text { each WBC, use SVM to } \\
\text { discern basophil and } \\
\text { eosinophil, CNN to } \\
\text { extract features from other } \\
\text { types of WBC }\end{array}$ & $\begin{array}{l}\text { WBCees, and it sometimes } \\
\text { considers a few non WBCs } \\
\text { as WBCs }\end{array}$ & $92.8 \%$ & $6 \mathrm{Acc}$ & curac & & & & \\
\hline & & Classification & Random forest & & & & & & & & \\
\hline $\begin{array}{l}\frac{\text { Khash }}{\text { man \& }} \\
\frac{\text { Abbas, }}{2013} \\
{[9]}\end{array}$ & $\begin{array}{l}\text { Method to } \\
\text { extract data from } \\
\text { microscopic cell } \\
\text { images without } \\
\text { performing } \\
\text { segmentation }\end{array}$ & Preprocessing & $\begin{array}{l}\text { Crop single cell images, } \\
\text { Otsu's thresholding, } \\
\text { median filter, edge } \\
\text { detection(canny operator), } \\
\text { pattern averaging }(2 \times 2 \\
\text { kernel) }\end{array}$ & $\begin{array}{l}\text { Merits } \\
\text { Reduce computational and } \\
\text { time cost without } \\
\text { performing segmentation } \\
\text { and extraction of local } \\
\text { feature from the blood cell }\end{array}$ & $\begin{array}{l}\text { Accur } \\
\text { Learn } \\
\text { Learn } \\
\text { Learn }\end{array}$ & $\begin{array}{l}\text { racy } \\
\text { ing } s \\
\text { ing } \\
\text { ing }\end{array}$ & $\begin{array}{l}\text { Scher } \\
\text { Scher } \\
\text { Scher }\end{array}$ & $\begin{array}{l}\text { me I } \\
\text { me I } \\
\text { me I }\end{array}$ & $\begin{array}{l}\text { I } 90 \% \\
\text { II } 80^{\circ} \\
\text { III } 75\end{array}$ & $\begin{array}{l}\%, \\
\% .1 \%\end{array}$ & \\
\hline & & Segmentation & - & images & \begin{tabular}{|l|} 
Leaming \\
Scheme \\
\end{tabular} & $\begin{array}{l}\text { Training A } \\
\text { Rater } \\
\mathrm{NBC}^{2}\end{array}$ & 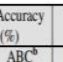 & $\begin{array}{l}\text { Testing. A } \\
\text { Rule } \\
\text { BC PI }\end{array}$ & $\begin{array}{l}\text { Aceuracy } \\
\text { te(n) } \\
A B C\end{array}$ & & 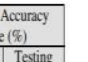 \\
\hline & classifier to & Feature & - & & LSS-I & $\begin{array}{c}\frac{A B C}{30130)} \\
100\end{array}$ & $\begin{array}{c}A B C-130) \\
100\end{array}$ & $\begin{array}{c}\frac{19 \mathrm{BC}}{(9 / 10)} \\
90\end{array}$ & (9/10) 90 & 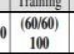 & \begin{tabular}{|c|} 
lesing \\
$(1820)$ \\
90
\end{tabular} \\
\hline & identify normal & Extraction & & & LS.II & $(202020)$ & $\frac{(2020)}{(200)}$ & $\begin{array}{lll}(15 / 520) \\
75\end{array}$ & \begin{tabular}{|l|}
$(17220)$ \\
85
\end{tabular} & $\begin{array}{cc}(40) / 40) \\
100\end{array}$ & $(32440) 80$ \\
\hline & and abnormal & Classification & ANN with 3 learning & & LS-III & $\frac{100}{(1010)}(100)$ & $\frac{100}{101010)}$ & $\frac{1301}{(22730)}$ & \begin{tabular}{|l|}
83 \\
$(2230)$ \\
76.6
\end{tabular} & $\begin{array}{l}120020) \\
1200\end{array}$ & $\begin{array}{ll}(45160) \\
75.1\end{array}$ \\
\hline & blood cells & & $\begin{array}{l}\text { schemes. LS1, LS2 and } \\
\text { LS3 }\end{array}$ & & & & & & & & \\
\hline
\end{tabular}


Ashwini P. Patil et al., International Journal of Emerging Trends in Engineering Research, 8(10), October 2020, 7320 - 7327

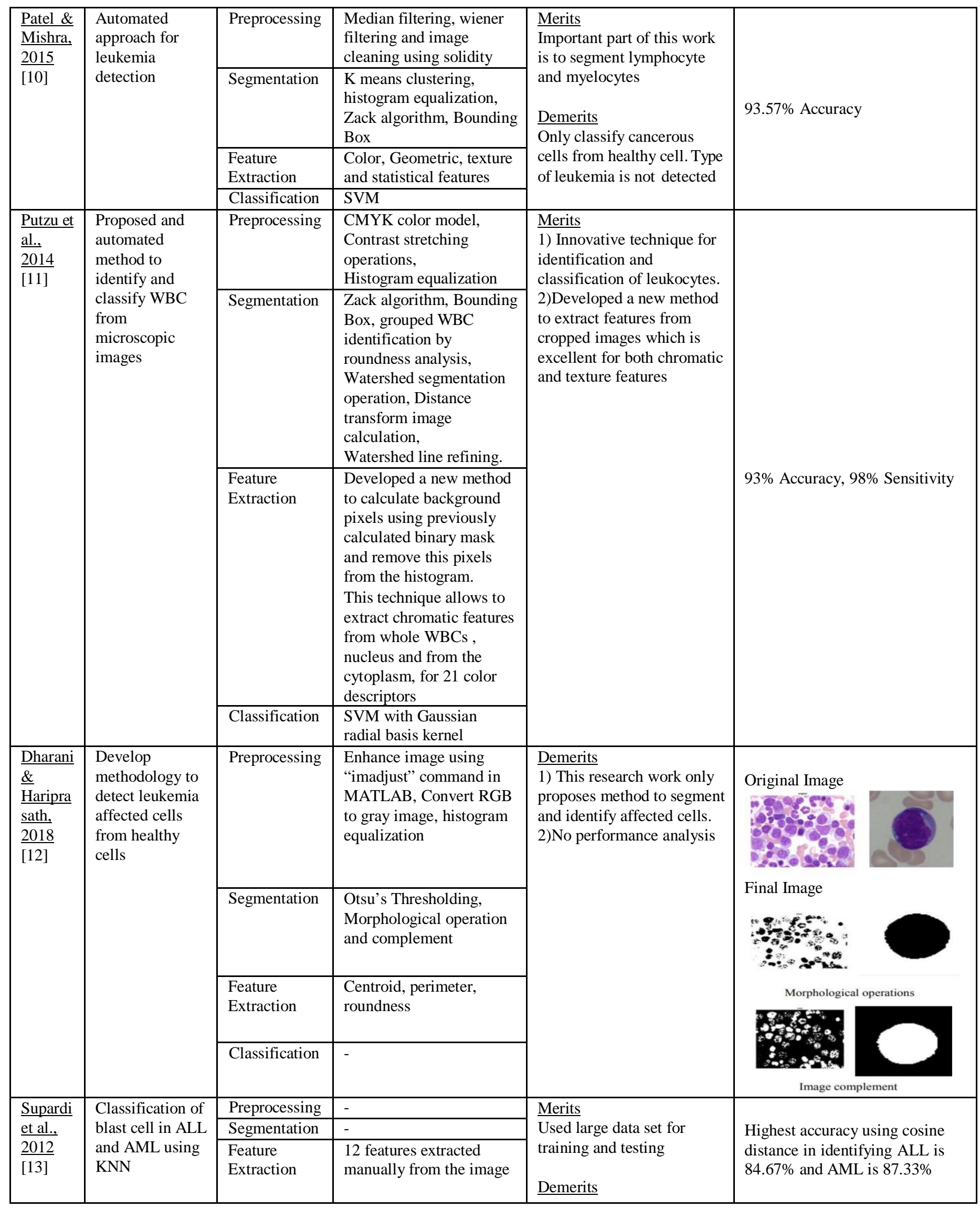


Ashwini P. Patil et al., International Journal of Emerging Trends in Engineering Research, 8(10), October 2020, 7320 - 7327

\begin{tabular}{|c|c|c|c|c|c|}
\hline & & Classification & KNN & $\begin{array}{l}\text { It is not a fully automated } \\
\text { approach. Features are } \\
\text { manually extracted }\end{array}$ & \\
\hline \multirow{4}{*}{$\frac{\frac{\text { Negm }}{\text { et al., }}}{\frac{2018}{[14]}}$} & \multirow{4}{*}{$\begin{array}{l}\text { Decision support } \\
\text { system that } \\
\text { comprises of } \\
\text { panel selection, } \\
\text { segmentation } \\
\text { using k-means } \\
\text { clustering } \\
\text { feature } \\
\text { extraction and } \\
\text { image } \\
\text { refinement to } \\
\text { identify } \\
\text { leukemia cells }\end{array}$} & Preprocessing & Select green component & \multirow{4}{*}{$\frac{\text { Merit }}{\text { High accuracy }}$} & \multirow{4}{*}{$\begin{array}{l}\text { Accuracy is } 99.517 \% \\
\text { Sensitivity is } 99.348 \% \\
\text { Specificity is } 99.529 \%\end{array}$} \\
\hline & & Segmentation & $\begin{array}{l}\text { K means algorithm, data } \\
\text { normalization }\end{array}$ & & \\
\hline & & $\begin{array}{l}\text { Feature } \\
\text { Extraction }\end{array}$ & WEKA tool & & \\
\hline & & Classification & ANN and decision tree & & \\
\hline \multirow{4}{*}{$\frac{\text { Mishra }}{\frac{\text { et al., }}{2017}}$} & \multirow{4}{*}{$\begin{array}{l}\text { ALL detection } \\
\text { using (MBS) } \\
\text { marker-based } \\
\text { segmentation, } \\
\text { (GLCM) gray } \\
\text { level } \\
\text { co-occurrence } \\
\text { matrix based } \\
\text { feature } \\
\text { extraction, and } \\
\text { (PPCA) } \\
\text { probabilistic } \\
\text { principal } \\
\text { component } \\
\text { analysis based } \\
\text { feature } \\
\text { reduction. }\end{array}$} & Preprocessing & $\begin{array}{l}\text { Histogram equalization, } \\
\text { wiener filtering }\end{array}$ & \multirow{4}{*}{$\begin{array}{l}\text { Merits } \\
\text { Proposed new technique for } \\
\text { identification and } \\
\text { classification of } \\
\text { lymphoblast }\end{array}$} & \multirow{4}{*}{$\begin{array}{l}\text { Accuracy } \\
\text { Segmentation } 96.29 \%, \\
\text { Classification } 99.004 \% \text { and } \\
\text { nucleus and cytoplasm } 96 \%\end{array}$} \\
\hline & & Segmentation & $\begin{array}{l}\text { marker-based } \\
\text { segmentation (MBS) }\end{array}$ & & \\
\hline & & $\begin{array}{l}\text { Feature } \\
\text { Extraction }\end{array}$ & GLCM and PPCA & & \\
\hline & & Classification & Random Forest & & \\
\hline \multirow[t]{4}{*}{$\frac{\frac{\text { Muntas }}{\text { a \& }}}{\frac{\text { Yusuf, }}{\frac{2019}{[16]}}}$} & \multirow[t]{4}{*}{$\begin{array}{l}\text { A detection } \\
\text { model for ALL } \\
\text { using principal } \\
\text { object } \\
\text { characteristics of } \\
\text { the color image }\end{array}$} & Preprocessing & $\begin{array}{l}\text { Separate the image into } \\
\text { Red, Green, Blue } \\
\text { channels. } \\
\text { Image enhancement, } \\
\text { Merge all } 3 \text { Channel } \\
\text { images and convert RGB } \\
\text { to HSV channel }\end{array}$ & \multirow{4}{*}{$\begin{array}{l}\text { Merits } \\
\text { Proposed system has two } \\
\text { novelties: 1) image } \\
\text { enhancement using } 3 \text { color } \\
\text { channels. (red green blue), } \\
\text { 2) feature extracted using } \\
\text { Densitometry components } \\
\text { extension. } \\
\text { Demerits } \\
\text { Main focus is on image } \\
\text { enhancement and feature } \\
\text { extraction. This work do not } \\
\text { include classification of } \\
\text { ALL and its subtypes }\end{array}$} & \multirow{4}{*}{$\begin{array}{l}81.54 \% \text { accuracy for Euclidean } \\
\text { Distance, } 76.92 \% \text { accuracy for } \\
\text { Manhattan, Canberra, } \\
82.31 \% \text { accuracy for Chebyshev }\end{array}$} \\
\hline & & Segmentation & $\begin{array}{l}\text { Filtering(Hue channel, } \\
\text { MatMask), local entropy } \\
\text { threshold, Morphological } \\
\text { operations, color image } \\
\text { segmentation }\end{array}$ & & \\
\hline & & $\begin{array}{l}\text { Feature } \\
\text { Extraction }\end{array}$ & $\begin{array}{l}\text { Feature extraction using } \\
\text { Energy (EN), Shanon } \\
\text { Entropy HX, Entropy(EP), } \\
\text { Log Energy Entropy (EE), } \\
\text { Variance (VA), Mean } \\
\text { (ME), and Correlation } \\
\text { (CO), Normalize features, } \\
\text { calculate similarity } \\
\text { measurement using } \\
\text { Euclidean Distance, } \\
\text { Manhattan, Canberra, and } \\
\text { Chebyshev methods }\end{array}$ & & \\
\hline & & Classification & - & & \\
\hline $\begin{array}{l}\text { Pathira } \\
\text { ge et al.. }\end{array}$ & $\begin{array}{l}\text { Image } \\
\text { processing }\end{array}$ & Preprocessing & $\begin{array}{l}\text { Noise removal, edge } \\
\text { detection }\end{array}$ & & \\
\hline
\end{tabular}


Ashwini P. Patil et al., International Journal of Emerging Trends in Engineering Research, 8(10), October 2020, 7320 - 7327

\begin{tabular}{|c|c|c|c|c|c|}
\hline \multirow{3}{*}{$\begin{array}{l}2016 \\
{[20]}\end{array}$} & \multirow{3}{*}{$\begin{array}{l}\text { techniques to } \\
\text { count RBC, } \\
\text { WBC and } \\
\text { platelet cells and } \\
\text { classify ALL and } \\
\text { CLL }\end{array}$} & Segmentation & Circular Hough transform & & \\
\hline & & $\begin{array}{l}\text { Feature } \\
\text { Extraction }\end{array}$ & $\begin{array}{l}\text { Geographical and color } \\
\text { based feature extraction }\end{array}$ & & \\
\hline & & Classification & - & & \\
\hline \multirow{4}{*}{$\begin{array}{l}\frac{\text { Shafiqu }}{\text { e et al., }} \\
\frac{2019}{[17]}\end{array}$} & \multirow{4}{*}{$\begin{array}{l}\text { Develop } \\
\text { automated } \\
\text { system for Acute } \\
\text { Lymphoblastic } \\
\text { leukemia } \\
\text { detection }\end{array}$} & Preprocessing & $\begin{array}{l}\text { RGB image converted to } \\
\text { CMYK color space, } \\
\text { Histogram equalization }\end{array}$ & \multirow{4}{*}{$\begin{array}{l}\text { Demerits } \\
\text { Only classification of blast } \\
\text { cell and normal cell. Do not } \\
\text { include ALL subtype } \\
\text { detection. } \\
\text { No novelty in this work }\end{array}$} & \multirow{4}{*}{$\begin{array}{l}93 \% \text { Accuracy } \\
92 \% \text { sensitivity } \\
91 \% \text { specificity }\end{array}$} \\
\hline & & Segmentation & $\begin{array}{l}\text { Zack algorithm, } \\
\text { watershed segmentation, } \\
\text { morphological operations }\end{array}$ & & \\
\hline & & $\begin{array}{l}\text { Feature } \\
\text { Extraction }\end{array}$ & $\begin{array}{l}\text { Color, shape and texture } \\
\text { features }\end{array}$ & & \\
\hline & & Classification & SVM & & \\
\hline \multirow[t]{4}{*}{$\frac{\text { Rawat }}{\frac{\text { et al., }}{2017}}$} & \multirow{4}{*}{$\begin{array}{l}\text { Develop system } \\
\text { to identify } \\
\text { leukemic cells } \\
\text { from healthy } \\
\text { cells and classify } \\
\text { them into ALL } \\
\text { and AML and } \\
\text { further classify } \\
\text { them into their } \\
\text { subtypes }\end{array}$} & Preprocessing & $\begin{array}{l}\text { Nucleus sub image } \\
\text { selection, } \\
\text { Histogram equalization, } \\
\text { Order statistic filter }\end{array}$ & \multirow{4}{*}{$\begin{array}{l}\text { Merits } \\
\text { 1)Classification of leukemia } \\
\text { into ALL, AML and their } \\
\text { subtypes with high accuracy } \\
\text { 2)Used genetic algorithm } \\
\text { for feature selections }\end{array}$} & \multirow{4}{*}{$\begin{array}{l}\text { Acute lymphoblastic cells, Acute } \\
\text { myeloid cells and healthy cells } \\
\text { classification accuracy is } 99.5 \% \text {, } \\
\text { Classification accuracy for L1, L2 } \\
\text { and L3 is } 97.1 \% \text {, } \\
\text { Classification accuracy for M2, } \\
\text { M3 and M5 is } 98.5 \% \text {. }\end{array}$} \\
\hline & & Segmentation & $\begin{array}{l}\text { Otsu's thresholding, } \\
\text { morphological opening, } \\
\text { image refining(hole filling } \\
\text { and border cleaning) }\end{array}$ & & \\
\hline & & $\begin{array}{l}\text { Feature } \\
\text { Extraction }\end{array}$ & $\begin{array}{l}\text { shape features, GLRL, } \\
\text { FOS, GLDS, NGTDM, } \\
\text { SFM, EDGE and GLCM, } \\
\text { signal processing based } \\
\text { features, transform } \\
\text { domain based models } \\
\text { (FPS and 2-D Gabor } \\
\text { transformation features } \\
\text { using three scales) and } \\
\text { color features. } \\
\text { Feature selection using } \\
\text { genetic algorithm }\end{array}$ & & \\
\hline & & Classification & $\begin{array}{l}\text { SVM with various kernel } \\
\text { functions. } \\
\text { Quadratic kernel, } \\
\text { Multilayer perceptron } \\
\text { (MLP) kernel, Linear } \\
\text { kernel, polynomial kernel } \\
\text { Gaussian Radial basis } \\
\text { function kernel }\end{array}$ & & \\
\hline
\end{tabular}

\section{RESEARCH ISSUES}

A lot of studies and research works are being conducted on automatic leukaemia detection. Many studies are achieving better accuracy and reliability, but researchers have to face a lot of challenges regarding dataset. Most of the research works do not use the same dataset which makes the comparison of accuracy and efficiency difficult. Low sample size and noise in the dataset are also some of the difficulties. Further challenges in this research area are extracting features from blood smear images to classify leukaemia into its various types and subcategories, hence researchers mostly focus on classifying cancerous cells and non-cancerous cells.

\section{CONCLUSION}

This review paper presents details of various methods and techniques, their merits and demerits which are summarized in a table. There is a lot of scope for future work in the development of automatic leukaemia detection system. Researchers should expand the size of their dataset and develop a system with high reliability and accuracy. Detection system should also be faster and economical. A lot of novel technologies could also be implemented to enhance the accuracy of segmentation and classification. Researchers should also focus on classifying leukaemia into various types and sub categories.

\section{REFERENCES}

1. J. M. Bennett et al., "Proposals for the Classification of the Acute Leukaemias French-American-British (FAB) 
Ashwini P. Patil et al., International Journal of Emerging Trends in Engineering Research, 8(10), October 2020, 7320 - 7327

Co-operative Group," British Journal of Haematology, vol. 33, no. 4. pp. 451-458, 1976, doi: 10.1111/j.13652141.1976.tb03563.x.

2. “Website.”[Online].Available:https://imagebank.hema tology.org/. [Accessed: 3-Oct-2020].

3. K. K. Jha and H. S. Dutta, "Mutual Information based hybrid model and deep learning for Acute Lymphocytic Leukemia detection in single cell blood smear images," Comput. Methods Programs Biomed., vol. 179, p. 104987, Oct. 2019.

4. R. Mohammed, O. Nomir, and I. Khalifa, "Segmentation of Acute Lymphoblastic Leukemia Using C-Y Color Space," International Journal of Advanced Computer Science and Applications, vol. 5, no. 11. 2014, doi: 10.14569/ijacsa.2014.051117.

5. R. Bhattacharjee and L. M. Saini, "Robust technique for the detection of Acute Lymphoblastic Leukemia," 2015 IEEE Power, Communication and Information Technology Conference (PCITC). 2015, doi: 10.1109/pcitc.2015.7438079.

6. R. Agrawal, S. Satapathy, G. Bagla, and K. Rajakumar, "Detection of White Blood Cell Cancer using Image Processing," 2019 International Conference on Vision Towards Emerging Trends in Communication and Networking (ViTECoN). 2019.

7. S. Mohapatra, D. Patra, and S. Satpathi, "Image analysis of blood microscopic images for acute leukemia detection," 2010 International Conference on Industrial Electronics, Control and Robotics. 2010, doi: 10.1109/iecr.2010.5720171.

8. J. Zhao, M. Zhang, Z. Zhou, J. Chu, and F. Cao, "Automatic detection and classification of leukocytes using convolutional neural networks," Med. Biol. Eng. Comput., vol. 55, no. 8, pp. 1287-1301, Aug. 2017.

9. A. Khashman and H. H. Abbas, "Acute Lymphoblastic Leukemia Identification Using Blood Smear Images and a Neural Classifier," Advances in Computational Intelligence. pp. 80-87, 2013, doi: 10.1007/978-3-64238682-4_10.

10. N. Patel and A. Mishra, "Automated Leukaemia Detection Using Microscopic Images," Procedia Computer Science, vol. 58. pp. 635-642, 2015.

11. L. Putzu, G. Caocci, and C. Di Ruberto, "Leucocyte classification for leukaemia detection using image processing techniques," Artif. Intell. Med., vol. 62, no. 3, pp. 179-191, Nov. 2014.

12. T. Dharani and S. Hariprasath, "Diagnosis of Leukemia and its types Using Digital Image Processing Techniques," 2018 3rd International Conference on Communication and Electronics Systems (ICCES). 2018, doi: 10.1109/cesys. 2018.8724075.

13. N. Z. Supardi, M. Y. Mashor, N. H. Harun, F. A. Bakri, and R. Hassan, "Classification of blasts in acute leukemia blood samples using k-nearest neighbour," 2012 IEEE 8th International Colloquium on Signal Processing and its Applications. 2012, doi: 10.1109/cspa.2012.6194769.
14. A. S. Negm, O. A. Hassan, and A. H. Kandil, "A decision support system for Acute Leukaemia classification based on digital microscopic images," Alexandria Engineering Journal, vol. 57, no. 4. pp. 2319-2332, 2018, doi: 10.1016/j.aej.2017.08.025.

15. S. Mishra, B. Majhi, P. K. Sa, and L. Sharma, "Gray level co-occurrence matrix and random forest based acute lymphoblastic leukemia detection," Biomedical Signal Processing and Control, vol. 33. pp. 272-280, 2017.

16. A. Muntasa and M. Yusuf, "Modeling of the Acute Lymphoblastic Leukemia Detection based on the Principal Object Characteristics of the Color Image," Procedia Computer Science, vol. 157. pp. 87-98, 2019, doi: 10.1016/j.procs.2019.08.145.

17. S. Shafique, S. Tehsin, S. Anas, and F. Masud, "Computer-assisted Acute Lymphoblastic Leukemia detection and diagnosis," 2019 2nd International Conference on Communication, Computing and Digital systems (C-CODE). 2019.

18. J. Rawat, A. Singh, H. S. Bhadauria, J. Virmani, and J. S. Devgun, "Computer assisted classification framework for prediction of acute lymphoblastic and acute myeloblastic leukemia," Biocybernetics and Biomedical Engineering, vol. 37, no. 4. pp. 637-654, 2017.

19. Joshi, M D et al. "White Blood Cells Segmentation and Classification to Detect Acute Leukemia Ms." (2013).

20. Pathirage, Sachethana \& Marapana, Shavini \& Chandrananda, Supun \& Amarathunga, Nishika. (2016). Detection of Leukemia using Image Processing and Machine Learning. 10.13140/RG.2.2.18469.24804.

21. M. Singh, C. P. Prasad, T. D. Singh, and L. Kumar, "Cancer research in India: Challenges \& opportunities," Indian J. Med. Res., vol. 148, no. 4, p. 362, Oct. 2018. 